\title{
Productivity and quality performance of an innovative firewood processor
}

\author{
Raffaele Cavalli, Stefano Grigolato, Andrea Sgarbossa \\ Department of Land, Environment, Agriculture and Forestry, University of Padova, Italy
}

\begin{abstract}
The growing interest about wood as fuel regards not only wood chips and pellets but also firewood, especially in mountain and rural areas where domestic heating plants are widely used. Due to the increased demand for firewood, harvesting activities have extended on broadleaved high forests as well as coppice. As a consequence, the diameter of logs has increased requiring larger and larger splitting machines; nowadays it is not uncommon to find on the market splitters able to process logs with diameter up to $50-60 \mathrm{~cm}$. In order to increase the productivity, the effort of machine producers is directed to obtain the complete splitting of the log into firewood in only one step using multiple ways splitting knives. This technical solution may cause some drawbacks especially when the splitting knives are not properly adapted to the log diameter; it happens that the size of firewood is not homogeneous and splinters are produced, which requires using screens to separate them from the main product. In order to evaluate the work quality of a firewood processor, equipped with multiple ways splitting knives, an experimental test has been carried out using a machine in which the log diameter is automatically detected through a laser device; according to the log diameter the multiple ways splitting knives (formed by fixed and mobile knives, the latter hydraulically operated) is properly set up to obtain regularly sized firewood. Furthermore the log is automatically centred on the splitting knife set-up. The results of the experimental test showed that the firewood processor is able to produce firewood with homogeneous size and with a low production of splinters, regardless of log diameter.
\end{abstract}

Correspondence: Raffaele Cavalli, Department of Land, Environment, Agriculture and Forestry (TESAF), University of Padova, viale dell'Università 16,35020 Legnaro (PD), Italy.

Tel.: +39.049.827.2724.

E-mail: raffaele.cavalli@unipd.it

Key words: biofuel, firewood, quality, productivity.

Contributions: RC, SG, AS, data collecting; SG, data analysis; AS, manuscript writing; RC, manuscript reviewing.

Conflict of interest: the authors declare no potential conflict of interest.

Received for publication: 6 February 2014.

Accepted for publication: 2 April 2014.

CCopyright R. Cavalli et al., 2014

Licensee PAGEPress, Italy

Journal of Agricultural Engineering 2014; XLV:228

doi:10.4081/jae.2014.228

This article is distributed under the terms of the Creative Commons Attribution Noncommercial License (by-nc 3.0) which permits any noncommercial use, distribution, and reproduction in any medium, provided the original author(s) and source are credited.

\section{Introduction}

The traditional technique of processing logs into smaller and handier firewood has been at the base of Countries development for centuries, as it represents a fundamental source of fuel for energy. The current increasing dynamism of the firewood market has led to the development and improvement of technologies able to process wood more efficiently by reducing consistently the time and labour required for firewood production (Lindroos, 2008; Loibneggar, 2011), as well as the risk of injuries for operators (Lindroos et al., 2008; Lindqvist and Nilsson, 2011). This was done by designing machinery with high productivity and ergonomics standards, able to process logs with a wide range of diameters and dimensions and maintaining as low as possible the production costs, even if the total capital investment became higher if related to simpler traditional methods.

The increased mechanisation in firewood production not always has been followed by an increased quality of the final product, especially regarding size homogeneity, since often, especially during the automated splitting phase, a big amount of splinters is produced. On the other hand, market and consumers have become very demanding regarding quality, especially for characteristics such as dimensions, wood species and water content (Casini, 1998; De Luca, 2004; Kärhä and Jouhiaho, 2009). With the development in 2010 of the European Standard EN 14961-1, defining specifications and classes of solid biofuels, a standard for firewood (EN 14961-5) has been introduced for the first time defining three different quality classes for firewood for non-industrial use: A1, A2 and B. Such a system will be able to allow producers and dealers to declare the quality of their goods, differentiating them in the market with a labelling scheme, while consumers' needs would be satisfied by a quality declared product (Kärhä and Jouhiaho, 2009).

The aim of this work is to study the productivity of a combined and automated saw and splitter processor and the quality of the firewood produced since a lack of studies about productivity and quality of such machinery (Manzone and Spinelli, 2014) is creating uncertainties on their technical application.

\section{Materials and methods}

\section{The firewood processor}

\section{Machine overview}

The firewood processor analysed in this study is the model S400 (Table 1), designed and built by the Officine Tollot Raffaello S.r.l. [Ponte Nelle Alpi (BL), Italy]. The experimental trial was carried out in June 2012 in Farra d'Alpago (Belluno province, Italy) at a biomass terminal for firewood production. The terminal is organised with a wide open storage space, able to host a significant amount of raw material. It allows whole logs to dry down to the required water content 
(dry and ready-to-use firewood production), and it acts as raw material buffer to avoid useless and costly processing stops during the production period (fresh firewood production).

The S400 is a firewood processor equipped with a log feeding deck capable of holding $20 \mathrm{t}$ of wood logs (approximately 28 logs: diameter $40 \mathrm{~cm}$, length: $6 \mathrm{~m}$ ) as maximum. The machine can be run both automatically or manually, as the operator can control every single operation. There are two control stations, specifically designed to improve the adaptability of the machine to every working condition. One is located on an upper floor that allows the operator to access and control each section of the machine during the production process. The other one is located on the side of the machine, nearby the final product conveyor, to allow the worker to operate a visual inspection on the product exiting from the splitting head. The feeding rack singularizes each log and moves it towards the feeding conveyor. Here optical devices measure the length of the log by means of photocells. The data are acquired and analysed by an electronic control unit that calculates the number and positions of cross cuts needed to obtain an average length of every single section and to ensure a regular dimension of the final product.

\section{Sawing}

The log is moved ahead to the sawing station, where a vertical hydraulic clamp comes down to firmly hold the log to be cut against four toothed rollers. A disc saw, hydraulically driven (blade diameter $120 \mathrm{~cm}$, kerf $9 \mathrm{~mm}$ ) can then operate the cross cut of each section at a predetermined length and let it to fall down into a V-shaped saddle mounted on a chain conveyor that moves it to the splitting station.

\section{Splitting}

A laser device measures the vertical diameter of the section and provides the control units with the data needed to lodge the piece in the centre of the splitting unit, where an auxiliary piston pushes and blocks the section on the splitting head. The splitting head is equipped with a fix cross knife, and other 12 multiple ways splitting knives, that are automatically adjusted on the basis of the diameter recorded, to optimise and regularise the dimension of the split pieces (Figure 1). Sections with a diameter less than $22 \mathrm{~cm}$ are split using only the fix cross knife (4 pieces). For diameters ranging from $22 \mathrm{~cm}$ to $27 \mathrm{~cm}$ four additional knives are lowered to operate (12 pieces). For diameters ranging from $27 \mathrm{~cm}$ to $33 \mathrm{~cm}$ no more knives are use, but a wider con-

Table 1. Technical characteristics of the firewood processor.

\begin{tabular}{lcc} 
& Unit & Value \\
Model & - & $\mathrm{S} 400$ \\
Weight & $\mathrm{t}$ & 11 \\
\hline Dimensions (W-L-H) & $\mathrm{m}$ & $8.5-7.5-2.6$ \\
Electric voltage & $\mathrm{V}$ & 380 \\
\hline Maximum electric power absorption & $\mathrm{kW}$ & 50 \\
Hydraulic pushing force & $\mathrm{kN}$ & 294 \\
\hline Hydraulic splitting circuit pressure & $\mathrm{MPa}$ & 20 \\
Auxiliary hydraulic circuit pressure & $\mathrm{MPa}$ & 8 \\
\hline Piston stroke time & $\mathrm{s}$ & 9 \\
Circular blade diameter & $\mathrm{cm}$ & 120 \\
\hline Circular blade kerf & $\mathrm{mm}$ & 9 \\
Max log diameter & $\mathrm{cm}$ & 40 \\
\hline Max splitting length & $\mathrm{cm}$ & 40 \\
Purchase price & $€$ & 75,000 \\
\hline
\end{tabular}

figuration of the present ones is set (12 pieces). Finally for diameters bigger than $33 \mathrm{~cm}$ the use of all 12 knives is required (20 pieces). Two parallel hydraulic pistons provide the max push force of $294 \mathrm{kN}$ needed to split the section into pieces passing through the knives. The high position of the splitting head allows the chops to fall down onto an inclined sieve that removes splinters occasionally produced. A belt conveyor then lifts firewood and releases it into the final storage.

\section{The experimental design}

The experimental test was divided in two parts. The first one was dedicated to the evaluation of the overall productivity of the equipment, while the second one was dedicated to measure the quality of the firewood produced.
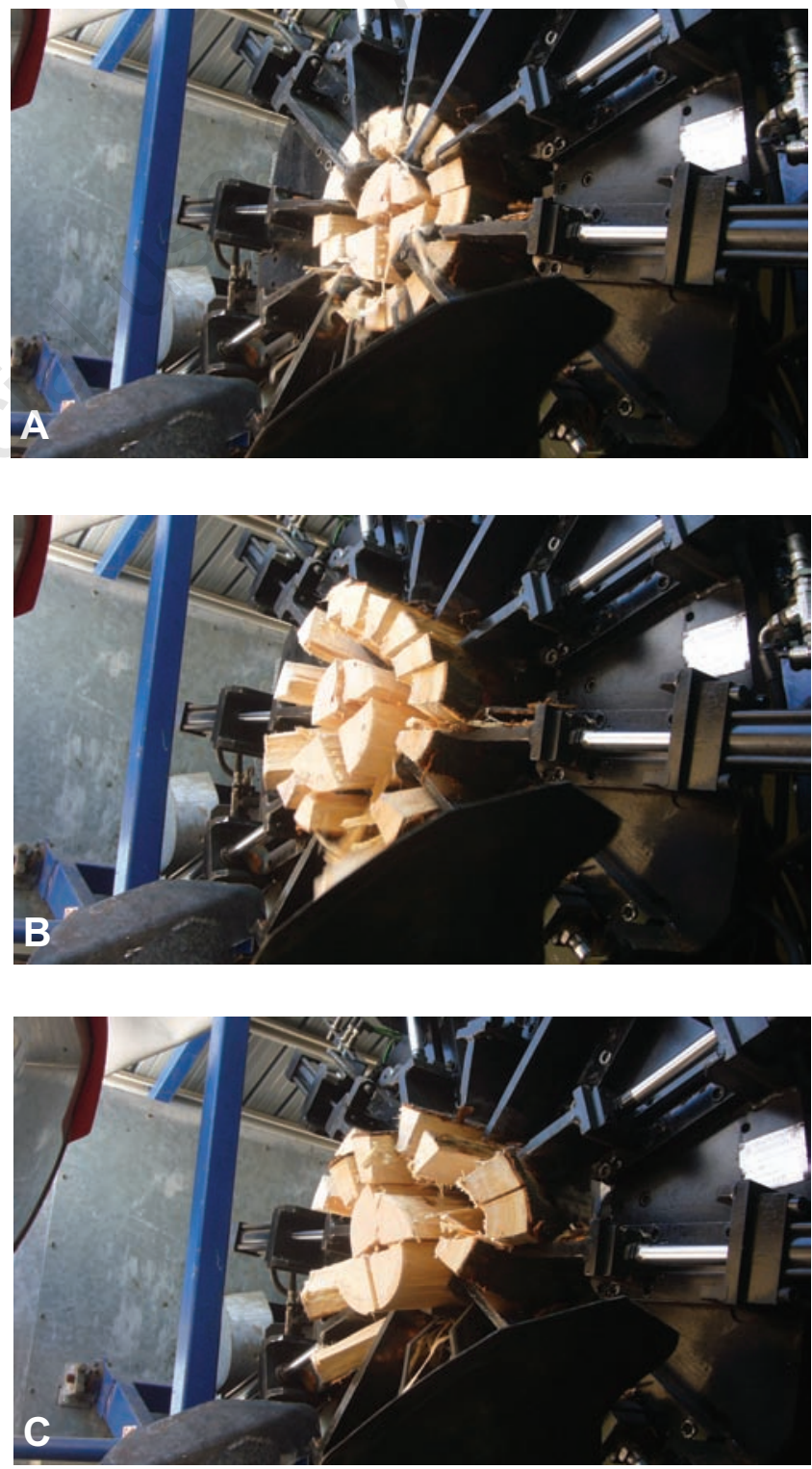

Figure 1. A-C) The splitting head in operation with 12 knives configuration. 


\section{Productivity}

For the productivity test, 15 fresh beech (Fagus sylvatica $\mathrm{L}$ ) logs were randomly selected from the raw material storage, their weight was determined on a weighbridge and loaded on the feeding rack regardless of the direction and tapering to avoid influencing productivity of the machine. For each log two perpendicular measures of the middle diameters and the total length of the piece were recorded for the later volume estimation. The total volume of the processed wood was $3.8 \mathrm{~m}^{3}$, the average diameter was $28 \mathrm{~cm}$ (SD 2.71) while the average log length was $4.05 \mathrm{~m}$ (SD 0.39) (Table 2).

Each log was marked with an identification number to make it recognisable during the processing operations. The operator was then asked to run the machine as usual, not being influenced by the experiment going on. A digital video camera was installed in the front of the machine, from where all the operations were visible. The recorded video was used in order to support the verification of the timing and recording activity by manual stopwatches. The time consumption of each producing phase was measured according to a time study layout adapted to the basic time concepts proposed by Magagnotti and Spinelli (2012). Two work phases were identified: work time (WT) and nonwork time (NWT).

The net productivity $\left(\mathrm{PMH}_{0}\right)$ of the work was obtained by dividing the total amount in terms of volume of firewood by the net working time, while the gross productivity $\left(\mathrm{PMH}_{\mathrm{t}}\right)$ was obtained by dividing the total amount of processed logs by the total working time.

\section{Quality}

For the quality test the operator loaded 11 beech logs on the feeding rack randomly selected from the raw material storage. The total volume of the processed wood was $2.7 \mathrm{~m}^{3}$, the average diameter was $26.4 \mathrm{~cm}$ (SD 4.4) while the average log length was $4.25 \mathrm{~m}$ (SD 0.64) (Table 3). Three people were involved in the test. One located near the control station measured and recorded the length and diameter of each section sawn, while other two measured the dimension of the pieces according to the limits imposed by the EN 14961-5 for the best quality of firewood for non-industrial use Al considering that the machine was set to produce L25 length class chops. To speed up the measuring operation a metal plate was prepared and provided with four round holes with the following diameters: $20 \mathrm{~mm} ; 50 \mathrm{~mm} ; 100 \mathrm{~mm} ; 150 \mathrm{~mm}$. Classes were defined as following: D2 $\leq 20 \mathrm{~mm}$; D5 $\leq 50 \mathrm{~mm}$; D10 $\leq 100 \mathrm{~mm}$; D15 $\leq 150$ $\mathrm{mm}$; D15+ >150 mm. Pieces belonging to Classes D2 and D5 are considered to be kindle quality. Each chop passing through a hole was considered to belong to that specific dimension class. If a chop was stuck in a hole, it was then removed and tested for the larger class. The chopping waste (i.e. splinters, sawdust, bark), that influenced negatively the productivity, was also measured. Small pieces of wood with a length shorter than the length of the pieces were not measured, and considered as waste. Some pieces of wood were randomly collected from the last conveyor belt, tightly sealed, and sent to the Biofuel Laboratory at Padova University where the moisture content was determined according to EN 14774:2009 standard and density according to EN 15150:2011 standard.

\section{Results and discussion}

\section{Time and productivity study}

The total running time was $1 \mathrm{~h}$ and $2 \mathrm{~min}$, during which the machine was able to process all the loaded logs $\left(3.8 \mathrm{~m}^{3}\right)$. This span time and volume processed is fully comparable with a number of other studies previously done, which results are summarised by Lindroos (2008). For what it concerns the time study, the distribution of the direct work time highlighted a $93.2 \%$ of main WT and $6.8 \%$ of NWT. The NWT was mainly due to stops of the process imposed by the machine safety system. The majority of them were due by jam of the feeding device, when the last section of a log was going to be processed. In that case, the operator has to move to the sawing station and reposition the section correctly on the cradle. Other case regards pieces that get stuck within the splitting head (the operator has to stop the automatic running of the machine and enlarge the knives configuration of the splitting head to let pieces fall down easily). The average time to process a section from the saw descent to the splitter piston return was $9 \mathrm{~s}$ and the two production phases (cut and split) take place simultaneously on two different sections.

The productivity study was based on the dependent variable net time (i.e. delays not included). The statistical analysis considered the development of a linear model with several independent variables, the evaluation of their non-linearity and the choice of the model by removing variables that were not significant and maintaining only the independent variables whose effect was significant at 0.05 level (length and diameter of $\log$ ). The regression linear model assumed the use of logarithmic transformed variables in order to express nonlinear models in linear model form. Therefore, the time consumption models were tested to obtain the best possible symmetrical distributions of residuals of the regression models and to achieve the best values for the coefficients. The statistical analysis was carried out using STATGRAPHICS ${ }^{\circledR}$ 16.1 (Statpoint Technologies, Inc., Warrenton, VA, USA).

The output shows the results of a model of multiple linear regression to describe the relationship between net processing time per log and the two independent variables: length and diameter. The equation of the fitted model resulted:

Time $\left[\mathrm{cmin} \log ^{-1}\right]=\operatorname{EXP}(3.3872+0.00213$ Length $[\mathrm{cm}]+$

Diameter $0.0623[\mathrm{~cm}]$ )

Since the P-value in ANOVA (Table 4 ) is less than 0.05 , there is a statistically significant relationship between the variables length and diameter at a confidence level of $95.0 \%$. The R-squared statistic indicates that the fitted model explains $90.8 \%$ of the variability in Time.

Table 2. Description of the main characteristics of the lot of logs for the productivity test.

\begin{tabular}{lcc} 
& Unit & Value \\
Total mass processed & $\mathrm{kg}$ & 3580 \\
Total volume processed & $\mathrm{m}^{3}$ & 3.8 \\
\hline Max diameter processed & $\mathrm{cm}$ & 33 \\
Max log length processed & $\mathrm{m}$ & 5.2 \\
\hline
\end{tabular}

Table 3. Description of the main characteristics of the lot of input logs for the quality study.

\begin{tabular}{lcc} 
& Unit & Value \\
Total volume processed & $\mathrm{m}^{3}$ & 2.7 \\
Wood density & $\mathrm{kg} / \mathrm{m}^{3}$ & 944.2 \\
\hline Moisture content & $\%$ & 55 \\
Maximum diameter processed & $\mathrm{cm}$ & 38.5 \\
\hline Maximum log length processed & $\mathrm{m}$ & 5.2 \\
\hline
\end{tabular}


Table 4. Result of the statistical analysis (confidence interval at 95.0\%).

\begin{tabular}{lccccc} 
Source & Sum of square & d.f. & Mean square & F & P-value \\
Length & 0.0853741 & 1 & 0.0853741 & 20.91 & 0.0006 \\
Diameter & 0.398263 & 1 & 0.398263 & 97.53 & 0.0000 \\
\hline Model & 0.483637 & 2 & & & \\
Parameter & Estimation & St. error & Lower limit & Upper limit & \\
Constant & 3.3872 & 0.253498 & 2.83482 & 3.93947 & \\
Length & 0.0021 & 0.00042 & 0.00119 & 0.00306 & \\
\hline Diameter & 0.0623 & 0.00630 & 0.04852 & 0.07599 & \\
\hline
\end{tabular}

The adapted R-squared statistical, which is most appropriate for comparing models with a different number of independent variables, was $89.3 \%$. The standard error of estimation shows that the standard deviation of the residues is 0.0639 .

The Durbin-Watson statistic evaluates the residues to determine if there is a significant correlation in the order in which they occur in the data file. Since the P-value is greater than 0.05 , there is no indication of serial autocorrelation in the residuals to the confidence level of $95.0 \%$.

Figure 2 shows the application of the productivity model. Four different representative log lengths were chosen to run the model, considering some of the common log lengths adopted in Italy for the harvesting of firewood logs $(640 \mathrm{~cm}-420 \mathrm{~cm}-320 \mathrm{~cm}-220 \mathrm{~cm})$. The function clearly shows how the productivity of the system increases with the diameter for all classes of length up to the maximum value reached at $32 \mathrm{~cm}$. After that, increasing diameters values reflect a decreasing trend in the total productivity of the machine. The ascending branch of the curve can be explained considering that the average section processing time ( $9 \mathrm{~s}$ ) depends on the piston speed in the splitting station, and not on the size of the section to be split, while the volume and numbers of pieces (that affects the productivity positively) are a direct function of the incoming diameter. On the other hand, diameters bigger than $32 \mathrm{~cm}$ (max diameter to be processed $40 \mathrm{~cm}$ ), involve the descent of all the multiple way knives available (12). Sometimes this fact results in a slowing down of the splitting process because of the increased friction between the knives and the wood. From the model it is also possible to notice that the total length of the incoming log is a key factor. The productivity in fact, increases considerably by running the machine with longer logs, because the time required to position the $\log$ in the feeding conveyor, during which the production stops (saw and split), is distributed over a greater volume processed. This trend is verified for log length ranging from $210 \mathrm{~cm}$ to $420 \mathrm{~cm}$, but is inverted when considering higher values. In the model in fact the productivity connected with logs $640 \mathrm{~cm}$ long is lower than the one calculated for $420 \mathrm{~cm}$ long logs. This is due to the increasing influence of the tapering of the incoming $\log$ that results in a certain number of sections with a small diameter, as a key factor affecting productivity. The productivity level registered for this machine seems to be comparable with what reported by Kährä and Jouhiaho (2009) even if the different setting and machine features lower the comparability of these results. This partly agrees with Manzone and Spinelli (2014) considering that steady levels of productivity are reported when machines are fed with pre-sorted or homogeneous log adapted to the machine needs.

\section{Quality of firewood}

Despite many local and regional preferences trends in choosing the appropriate firewood for domestic heating (Abbot et al., 1997; Lingens et al., 2005; Ramos et al., 2008), the parameters of firewood that have

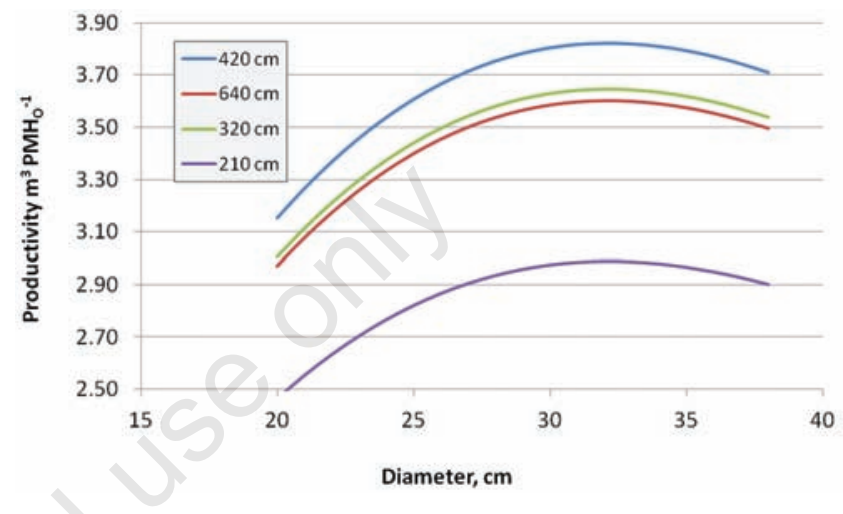

Figure 2. Productivity model according to mean diameter and length of the input log.

to be observed to define the overall quality are given in the EN 14961-5 standard, through which firewood for non-industrial use is classified. This standard refers to three different quality classes, on the basis of decreasing measured parameters: $\mathrm{A} 1, \mathrm{~A} 2$ and $\mathrm{B}$. The analysis of data about the firewood cross-section size, shown in Figure 3, reveals that $92 \%$ of pieces produced are included in D10 and D15 classes, which are the ones recommended for high quality A1 firewood. A subsequent reselection of the product to separate D10 from D15 pieces does not seem to be effective from an end-user perspective, and, as a consequence, seems not to be convenient, as soon as the quality remains so high. Classes D2 and D5 do not exceed 5\%, and do not represent an issue for quality and price of the firewood. In the case that the presence of undersized particles is a key factor for the commercialisation of the product, they can be easily removed from the load, packaged and sold separately as kindle. On the other hand, the presence of oversized pieces (D15+) often is considered as a quality-limiting factor for the end-user, especially when the firing appliances are small and domestic ones, where the size of the furnace is the limiting factor. In that case oversized pieces can be selected manually (directly by the machine operator) and reprocessed with an auxiliary splitting machine (vertical splitting wedge). Alternatively this material can be sorted out and sold as fireplace pieces.

The length of firewood is predetermined by the crosscut length and depends on the setting of the machine. The machine control unit, on the basis of the log length and the length class set by the operator, can adjust the length of each cut automatically to minimise the final loss. On the other hand the operator, on the basis of specific needs, can set the cut length at a fixed value. The results show that the firewood produced is within the length class L33 according to the EN 14961-5 standard. It means that more than $85 \%$ of pieces have a length less than 


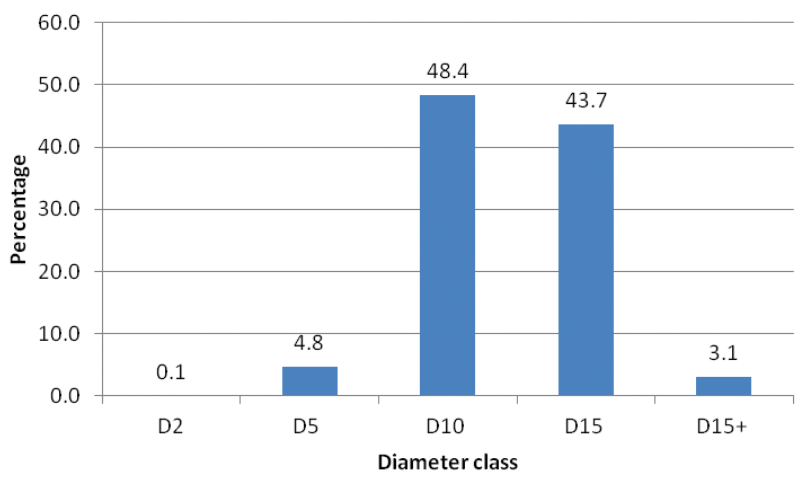

Figure 3. Distribution of piece diameter classes (EN 14961-5 standard).

$33 \pm 2 \mathrm{~cm}$, and no more than $15 \%$ of them are oversized. In the experiment measurements the average length of chucks was $25.15 \mathrm{~cm}$ and no pieces were longer than the limit value, proving that the automatic length setting is accurate enough to ensure a regular sized product (Figure 4). The process highlighted also a net production in mass of pieces of $95 \%$ of the total mass processed.

The average moisture content was estimated at $55 \%$ on dry basis. This value is important to be considered, as fresh logs could produce less splinters and the total energy required for their processing could be reduced. Moreover splitting whole logs to produce firewood increases the surface exposed to the air as well as the surface on volume ratio, thus facilitating the drying process (Erkkilä and Alakangas, 2008) and reducing the risk of decaying activity.

\section{Conclusions}

The increasing demand for firewood is driving a strong evolution in the organisation of the supply and production as well as the development of new technologies. New pieces of equipment are released every year to respond to the increasing needs of producers concerning productivity, operator safety and product quality. An innovative firewood processor, capable to adapt the cutting length and the splitting head settings to the $\log$ and section dimensions has been experimented in this study with the aim of evaluating productivity and product quality. Results show that the automatic reading of log lengths and the correct settings of the control unit are key factors to ensure regularity in cuts that are maintained within specific ranges of variability. The automatic selection of the splitting knives configuration is able to give consistency to the cross section dimension, adapting and optimising the number and volume of pieces produced at every run. On the other hand purchase costs are still fairly high, making the payback period longer if compared with other combined system with the same productivity. The high power required to drive all the hydraulic motors (approximately $50 \mathrm{~kW}$ ) could represent another limiting factor for the machine installation. The organisation of the working phases and supply logistics are fundamental to obtain high productivity levels and reduce delay times to the minimum.

The implementation of such technology on a commercial product and its further optimisation, especially regarding the operational speed, together with an appropriate seasoning of both feedstock and final product can lead to the meeting of the higher level of firewood quality as stated by the reference EN standard.

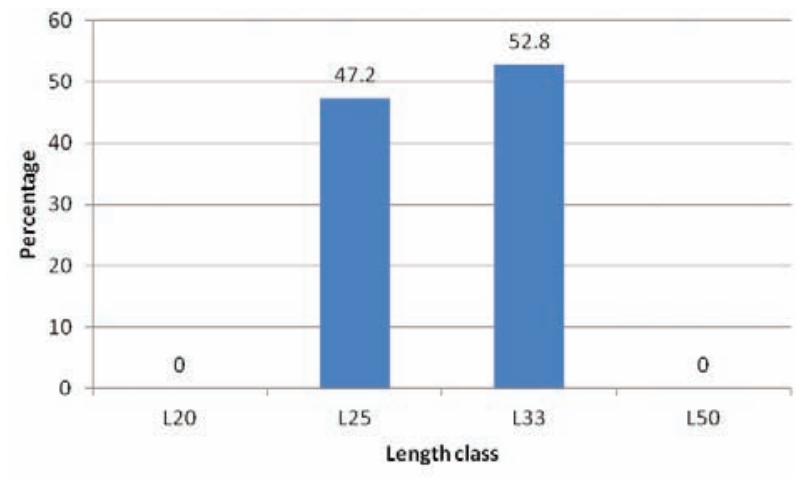

Figure 4. Distribution of piece length classes (EN14961-5 standard).

\section{References}

Abbot P., Lowore J., Khofi C., Werren M. 1997. Defining firewood quality: A comparison of quantitative and rapid appraisal techniques to evaluate firewood species from a southern African savanna. Biomass Bioenergy. 12:429-37.

Casini L. 1998. La legna da ardere in 10 punti (Explaining fuelwood in ten points). Sherwood. 4:35-41.

De Luca E. 2004. Approvvigionamento e commercializzazione della legna da ardere (Supply and selling firewood). Sherwood 10:21-3.

Erkkilä A., Alakangas E. 2008. Manual for firewood production. VTT-R11021-08. Available from http:/www.ruraldevelopment.org.uk/ northwoods/files/2012/12/Firewood-production-manual_EN.pdf Accessed: July 2012.

Kährä K., Jouhiaho A. 2009. Producing chopped firewood with firewood processors. Biomass Bioenergy. 33:1300-9.

Lindqvist A., Nilsson 0. 2011. Hand injury from powered wood splitters: machine safety, patterns of use and injury events. Int. J. Occup. Safety Ergon. 17:175-86.

Lindroos 0. 2008. The Effects of increased mechanization on time consumption in small-scale firewood processing. Silva Fennica 42:791-805.

Lindroos 0., Aspam E.W., Lidestav G., Neely G. 2008. Accidents in family's firewood production, Accident Anal. Prevent. 40:877-86.

Lingens A., Windeisen E., Wegener G. 2005. Investigating the combustion behavior of various wood species via their fire gases. Wood Sci. Technol. 30:49-60.

Loibneggar T. 2011. Roadmap for implementing standards. Available from: http://www.biomassenergycentre.org.uk/pls/portal/docs/PAGE /PRACTICAL/FUEL_SUPPLY/STANDARDS/ROADMAP\%20FOR\%20I MPLEMENTING\%20STANDARDS.PDF Accessed: July 2012.

Magagnotti N., Spinelli R. 2012. Good practice guidelines for biomass production studies. COST Action FP-0902. CNR IVALSA, Florence, Italy.

Manzone M., Spinelli R. 2014. Efficiency of small-scale firewood processing operations in Southern Europe. Fuel Process. Technol. 122:58-63.

Ramos M., Medeiros P., Almeida A., Feliciano A., Albuquerque U. 2008. Can wood quality justify local preferences for firewood in an area of caatinga (dryland) vegetation?. Biomass Bioenergy. 32:503-9. 